\title{
ACE 28
}

\section{TENNESSEE VALLEY AUTHORITY: UNA EXPERIENCIA DE PLANIFICACIÓN TERRITORIAL MODÉLICA}

Miquel Corominas y Jordi Franquesa

Cómo citar este artículo: COROMINAS, M. y FRANQUESA, J. Tennessee Valley Authority: una experiencia de planificación territorial modélica [en línea] Fecha de consulta: dd-mm-aa. En: ACE: Architecture, City and Environment = Arquitectura, Ciudad y Entorno, 10 (28): 11-32, 2015. DOI: 10.5821/ace.10.28.3935. ISSN: 1886-4805.

\section{ACE




\title{
ACE 28
}

Electronic offprint

Separata electrónica

\section{TENNESSEE VALLEY AUTHORITY: AN EXEMPLARY EXPERIENCE OF TERRITORIAL PLANNING}

Key words: Tennessee Valley Authority; territory planning; new settlements; recovery.

\begin{abstract}
After the great depression derived from the 1929 crack, the Government of Franklin Roosevelt implemented a territorial initiatives without any precedent. The creation of the Tennessee Valley Authority at the end of the 30' implied a recovery of an extense territory punished and impoverished by the unstable floods, but at the same time implied also a first step for the country's economic recovery obtaining a great potential energy survey, showing in turn an efficient capacity of management of the territory that meant new urban settlements, new cultural and entertainment activities, new dinamic economies, and new hopes. The present work wants to make a deph look to this procedure to assess its main contributions and recognize its territorial repercussions on a current key.
\end{abstract}

\section{ACE




\title{
TENNESSEE VALLEY AUTHORITY: UNA EXPERIENCIA DE PLANIFICACIÓN TERRITORIAL MODÉLICA
}

\author{
COROMINAS, Miquel ${ }^{1}$ \\ FRANQUESA, Jordi
}

Remisión inicial: 25-02-2013

Remisión final: 19-02-2014

Palabras clave: Tennessee Valley Authority; planificación territorial; nuevos asentamientos; recuperación.

\section{Resumen}

Después de la gran depresión derivada del gran crack de 1929, el gobierno de Franklin D. Roosevelt llevó a cabo una iniciativa de carácter territorial sin precedentes. La creación de la Tennessee Valley Authority a finales de los años 30 implicó la recuperación con creces de un vastísimo territorio castigado y empobrecido por las inestables inundaciones, pero al mismo tiempo supuso también un primer paso para la recuperación económica del país mediante la obtención de un importantísimo potencial energético, demostrando a su vez una eficiente capacidad de gestión del territorio que se tradujo en la creación de nuevos asentamientos urbanos, nuevas actividades culturales y de ocio, nuevas dinámicas económicas, y nuevas y firmes esperanzas. El presente trabajo pretende hacer una mirada atenta a este proceso para poder valorar sus principales aportaciones y reconocer sus repercusiones territoriales en clave actual.

\section{Introducción}

El final de la primera guerra mundial abre para Estados Unidos un periodo dominado por los ideales de expansión productiva y de prosperidad económica, aseguradas por el grueso de los inversores y por los grupos de poder. La negativa del Senado norteamericano a ratificar el pacto de la Sociedad de Naciones ${ }^{2}$ elimina los lazos y vínculos de solidaridad con Europa. En este periodo, dentro de este escenario de aislamiento político se abocaría definitivamente a los principios del liberalismo económico, capaz de impedir cualquier interferencia del Estado en los asuntos del mundo privado, aprovechando los grandes progresos de la ciencia y de la técnica.

Miquel Corominas: Catadràtic del Departament d'Urbanisme i Ordenació del Territori, Escola Tècnica Superior d'Arquitectura de Barcelona. Email de contacte: miquel.corominas@upc.edu

Jordi Franquesa: Professor del Departament d'Urbanisme i Ordenació del Territori, Escola Tècnica Superior d'Arquitectura de Barcelona. Email de contacte: jordi.franquesa@upc.edu

${ }^{2}$ El Pacto de la Sociedad de Naciones pretendía crear un nuevo organismo internacional con el fin de garantizar la paz entre los países tras la Primera Guerra Mundial. 
En este contexto de gran prosperidad económica y expansión productiva, la industria se ve tecnológicamente reforzada, y los nuevos empleos privados se multiplican, especialmente en el sector terciario. Las grandes empresas y los trusts financieros conquistan los mercados comerciales europeos y del continente americano, mientras que los sindicatos van perdiendo fuerza.

La distribución de la población en el territorio revela estos procesos. El censo de 1920 indica por primera vez que más del $50 \%$ de la población vive en núcleos urbanos de más de 2.500 habitantes. Por su parte, el número de trabajadores vinculados a las industrias manufactureras también aumenta considerablemente. En conjunto se asiste a una gran expansión urbana, como fenómeno de masas: con la producción del modelo Ford $\mathrm{T}^{3}$, el vehículo es ahora asequible a amplios estratos de población. De los 9 millones de unidades que circulan por las carreteras en 1920 se pasa a los 27 millones en 1930.

Todo ello supone un enorme impulso al crecimiento urbano periférico, ya sea por la alta accesibilidad que se permite en el hinterland, como por los efectos de congestión que provocan los downtowns. El crecimiento aparece ahora caracterizado por el típico fenómeno de la expansión discontinua, el leap frog developement ${ }^{4}$, a propósito de la transición desde los medios de transporte colectivos a los individuales. Los transportes de masa, en todas partes, experimentan una grave crisis económico-financiera y obligan a que las autoridades públicas releven estas actividades a los sectores privados.

La expansión de los suburbios acentúa la especialización de los usos y de las partes de la estructura urbana, incentivando la estratificación y la segregación residencial. Los centros urbanos fuertemente terciarizados experimentan y generalizan los crecimientos en altura, especialmente en Nueva York y Chicago, gracias a los avances de las técnicas constructivas. En los barrios residenciales se experimentan fuertes cambios en las tendencias a la estratificación étnica y de clase.

Pero sobre esta eufórica certidumbre de prosperidad que va a dominar toda la década de los años 20 cae de improviso la dramática crisis económica de octubre de 1929, y pone al desnudo las graves contradicciones del sistema social, financiero y productivo existente. Una desordenada política de créditos provocó una carrera febril de inversiones sin concierto alguno, y bajo las presiones especulativas, las acciones se elevaron muy por encima de la efectiva solidez patrimonial de las empresas. Los mismos fundamentos del sistema social se ponen en peligro y la tradicional confianza en el equilibrio automático del sistema se ve seriamente sacudida.

A partir de esta crisis, un episodio que no puede de ninguna manera equipararse a una de tantas crisis pasajeras y recurrentes del sistema, el país se abocaría a una gran depresión económica, lo cual va requerir una reelaboración profunda de las estructuras y de los métodos de dicho sistema. Los EE.UU., en los años 20 los abanderados del capitalismo mundial,

\footnotetext{
${ }^{3}$ El Ford T, un vehículo fabricado entre 1908 y 1927, supuso un cambio radical en los sistemas de producción, a la vez que supuso un fuerte impacto en términos de movilidad social y en la aparición de nuevas dinámicas territoriales.

${ }^{4}$ El leap frog developement, o literalmente, desarrollo del salto de la rana, es el nombre que se utiliza para calificar los crecimientos urbanos de bolsas de viviendas unifamiliares aisladas en Estados Unidos y que singularizan el territorio de las áreas periféricas de muchas de sus ciudades.
} 
ofrecen el aspecto de un país a la deriva, donde miles de inversores pierden sus ahorros, las fábricas y los bancos cierran sus puertas, y el desempleo aumenta vertiginosamente.

En 1932 la crisis toca fondo, con 13 millones de parados, y una producción inferior en un 55\% a la de 1929, y 32.000 quiebras. Los signos de la depresión son visibles en los campos, en las ciudades pequeñas y en las metrópolis, donde aumenta el número de personas sin hogar y donde muchas familias de clase obrera se ven obligadas a vivir en condiciones extremadamente precarias.

En el momento del estallido de la crisis, Herbert Hoover ocupaba la presidencia de los Estados Unidos. Hoover no fue inoperante, sino que trató de encauzar la crisis, una crisis que creyó pasajera. Sin embargo, la política llevada a cabo por Hoover fue un fracaso: el país se hundió en la recesión y las quiebras se multiplicaron.

Franklin Delano Roosevelt, ya era conocido por su labor como gobernador del estado de New York en los primeros años de crisis, con un programa de asistencia a los parados y de recuperación industrial. En los primeros años en la Casa Blanca, después de su elección como presidente en 1932, promulgó un conjunto de medidas políticas de carácter interno que se completaron con actos legislativos del Congreso para hacer frente a la crisis basada en el intervencionismo del estado. A esta labor se la conoce como New Deal y nos ofrece un modelo coherente de racionalización del capitalismo americano. Roosevelt realizó inversiones importantes, especialmente en obras públicas, y permitió el acceso a recursos financieros a través de las diversas agencias gubernamentales. Los resultados económicos fueron moderados, pero la situación mejoró. En este sentido, levantó la economía del estancamiento, se regeneró el poder adquisitivo de la población y fomentó la recuperación de las inversiones (Moore, 1967).

La Tennessee Valley Authority, es sin duda el proyecto emblemático del New Deal, una propuesta de recuperación de un extenso territorio multiestatal degradado mediante un complejo y coordinado sistema de desarrollo, que debía tener importantes repercusiones para todo el país.

Las realizaciones urbanísticas más importantes del New Deal se pueden resumir en las intervenciones vinculadas a la vivienda, los ambiciosos proyectos de las Green Belt cities, lideradas por la iniciativa de Rexford Guy Tugwell (con escasos resultados).

\section{Orígenes y gestión de la Tennessee Valley Authority}

El proyecto de la Tennessee Valley Authority fue el mayor logro del New Deal, y es donde se llevaron a cabo las ideas más radicales del Regional Planning Association of America (en adelante, RPAA), un grupo de técnicos arquitectos, economistas y técnicos que desde ya hacía muchos años defendían a ultranza las intervenciones regionalistas ${ }^{5}$. La Tennessee Valley

\footnotetext{
${ }^{5}$ En los estatutos de la Regional Planning Association of America, fundada en 1923 en New York por Clarence Stein y Lewis Mumford entre otros, ya figura el objetivo de mejorar la region deprimida del Valle del Tennessee.
} 
Authority (en adelante, TVA) supone una de las pocas experiencias de planificación territorial en los EE.UU., pero con unos resultados realmente relevantes (Huxley, 1944).

Así pues, pocos meses después de la toma de posesión, el 10 de abril de 1933, el presidente Roosevelt solicita del Congreso la aprobación de la constitución de la TVA, un organismo federal de planificación para todo el territorio de la cuenca del río Tennessee, cuya constitución es firmada el 18 de mayo.

Cuando se aprueba la Tennessee Valley Authority Act, el nuevo organismo supone la victoria del principio conforme el cual el gobierno federal tiene derecho a planificar y administrar las fuentes de energía $y$, por tanto, tiene competencias para poder aplicar políticas intervencionistas.

Junto al pequeño pantano de Muscle Shoals en Alabama se emplazó, en los años de la primera guerra mundial, una fábrica de nitrato sintético que se utilizaba tanto en la fabricación de explosivos como en la de fertilizantes. El Gobierno federal compró Muscle Shoals en 1916. La construcción de la presa comenzó en esta ubicación para producir energía para la fabricación de explosivos necesarios durante la primera Guerra Mundial, pero la guerra había concluido antes de que este nuevo proyecto operacional pudiera dar sus frutos. Durante la década de 1920, el Congreso consideró si la propiedad debe seguir siendo de carácter público, o ser vendida al sector privado.

Sin embargo, el coste de mantenimiento del dique respecto al rendimiento de la fábrica era excesivo, ya que requería ingentes cantidades de energía, por lo que se produjo un fuerte debate sobre la subvención del pantano para los fines industriales. La fábrica solicitó un préstamo al $5 \%$ de interés de 30 millones de dólares como anticipo. Al no serle concedido cerró durante varios años, y empezó una importante degradación del área. Esta confrontación sobre su operatividad se desarrolla entre los grupos progresistas y los más intransigentes detractores de la intervención estatal en la economía, en favor de la transferencia al capital privado de la presa y de las centrales eléctricas (Creese, 1990).

El origen del programa de la TVA es, primeramente, la respuesta directa que da el gobierno a las resistencias ofrecidas contra la utilización de las instalaciones de Muscle Shoals para la producción de energía eléctrica. Sin embargo, el programa de la TVA no se va a ceñir exclusivamente en la creación y administración de algunas instalaciones públicas: el propio presidente Roosevelt ya advierte claramente que el gobierno espera de la TVA una intervención global de la planificación que ha de ir mucho más allá de los ámbitos similares a Muscle Shoals, apuntando así sus repercusiones territoriales (Hargrove, 1984).

El senador George W. Norris fue, en realidad, el padre fundador de la TVA. Norris luchó para que la propiedad continuara bajo control público. Tuvo poco apoyo de sus colegas del Congreso, hasta la llegada de la depresión, pero el presidente Roosevelt apoyó en seguida el plan del senador Norris y lo incorporó como una forma de ayudar a lograr el éxito de su New Deal, destinado a salvar a la economía de Estados Unidos. Con la falta de inversión nacional en la región del Valle de Tennessee, el presidente Roosevelt pidió que el Congreso creara una corporación gubernamental, pero con una flexibilidad de iniciativa que fuera propia de una empresa privada. De esta manera, y liberada de los procesos tradicionales a los que se ven 
ligados los entes y órganos públicos gubernativos, la TVA se hace cargo del empleo de sus propios trabajadores, de los salarios y de las condiciones de trabajo, y desarrolla sus propios programas, pasando por encima de la subdivisión de competencias entre instituciones diversas, aunque no sin colaborar con los entes locales correspondientes. De esta manera, no pasa a depender de ningún departamento gubernamental existente, sino que depende directamente del Congreso y del presidente.

El consejo de dirección de la TVA se compone en sus inicios de tres miembros directivos y su estructura organizativa se divide en secciones:

- La planificación económica y social, liderada por David E. Lilienthal.

- La política agrícola y producción de fertilizantes, a cargo de Harcourt A. Morgan.

- La política energética, controlada por Arthur E. Morgan.

David Lilienthal fue de hecho la pieza clave para popularizar la imagen del TVA. Mediante una importante campaña de publicaciones de artículos de revista, películas, ensayos y exposiciones, Lilienthal convenció religiosamente al público de que el valle merecía una electricidad barata, sobre todo para sus agricultores rurales, que hasta entonces no habían sido servidos todavía por las compañías energéticas del sudeste. La TVA de Lilienthal iba a salvar el valle mediante la producción barata de energía. La agencia tenía múltiples ámbitos de competencia:

- El proyecto y construcción de los diques a lo largo del río.

- La adquisición de terrenos.

- La construcción de líneas de transporte eléctrico.

- La distribución y venta de la energía eléctrica producida en la cuenca.

- El control y regulación del flujo del río.

- El control del sistema de navegación y transporte por el río.

- La planificación y el proyecto de los nuevos asentamientos urbanos.

La TVA realizará por tanto intervenciones específicas de carácter territorial, como el control de las inundaciones, de la navegación fluvial que permitió utilizar barcos de mayor calado para el transporte de mercancías, la distribución de la energía eléctrica, el acondicionamiento del suelo, llevando a cabo también nuevas aplicaciones experimentales de algunas técnicas de actuación en planes de repoblación forestal, de asentamientos industriales y, por supuesto, de asentamientos humanos.

\section{3. Ámbito}

Los límites territoriales de la TVA corresponden a la propia cuenca fluvial del Tennessee, afluente del Mississippi, de unos $1.000 \mathrm{~km}$ de longitud y de una superficie de unos 100.000 $\mathrm{km}^{2}$, que se extiende a lo largo de 7 estados, incluyendo Carolina del Norte, Virginia, las zonas septentrionales de Georgia, de Alabama y de Mississippi y parte de Kentucky (ver Figura 1). En total se trata de una extensión equivalente a Gran Bretaña, con 4,5 millones de habitantes, 
circundada por algunas ciudades importantes como San Luís, Louisville, Atlanta o Birmingham, y muy especialmente Memphis, y con una red de ciudades intermedias como Nashville, Chattanooga o Knoxville. La región tenía, además, diversidad de climas, de recursos, de composición racial y de modelos culturales (Kyle, 1958).

Figura 1. El ámbito de intervención de la Tennessee Valley Authority

Fuente: Elaboración propia.

Pero lo más relevante del proyecto es su dimensión regional, una lectura que nunca se había alcanzado en términos de superficie hasta entonces. La política agrícola de ayudas y la política de construcción de industrias descentralizadas respecto a las grandes áreas metropolitanas facilitaban el acceso al área de nuevos inmigrantes, que seguían el viejo esquema americano de compartir el trabajo industrial con la agricultura (part-time-work) que el New Deal proponía como alternativa al paro, haciendo célebre la frase de Henry Ford ningún seguro es mejor que un trozo de tierra.

En este sentido, las instalaciones de Muscle Shoals constituyen tan sólo una pequeña parte de la unidad potencial de todo el río Tennessee. La propuesta trasciende los límites de la mera distribución de la energía, y abarca los vastos campos del control de las inundaciones, de la erosión del suelo, de la repoblación forestal, de la eliminación de las tierras marginales de la explotación agrícola y de la distribución y diferenciación de la industria. Todo ello conduce a la planificación nacional de toda una cuenca que abarca muchos estados y afecta a la vida y bienestar de millones de individuos. En términos de Lilienthal afecta y da vida a todas las formas de actividad humana (ver Figura 2). 
Figura 2. La región del valle del Tennessee

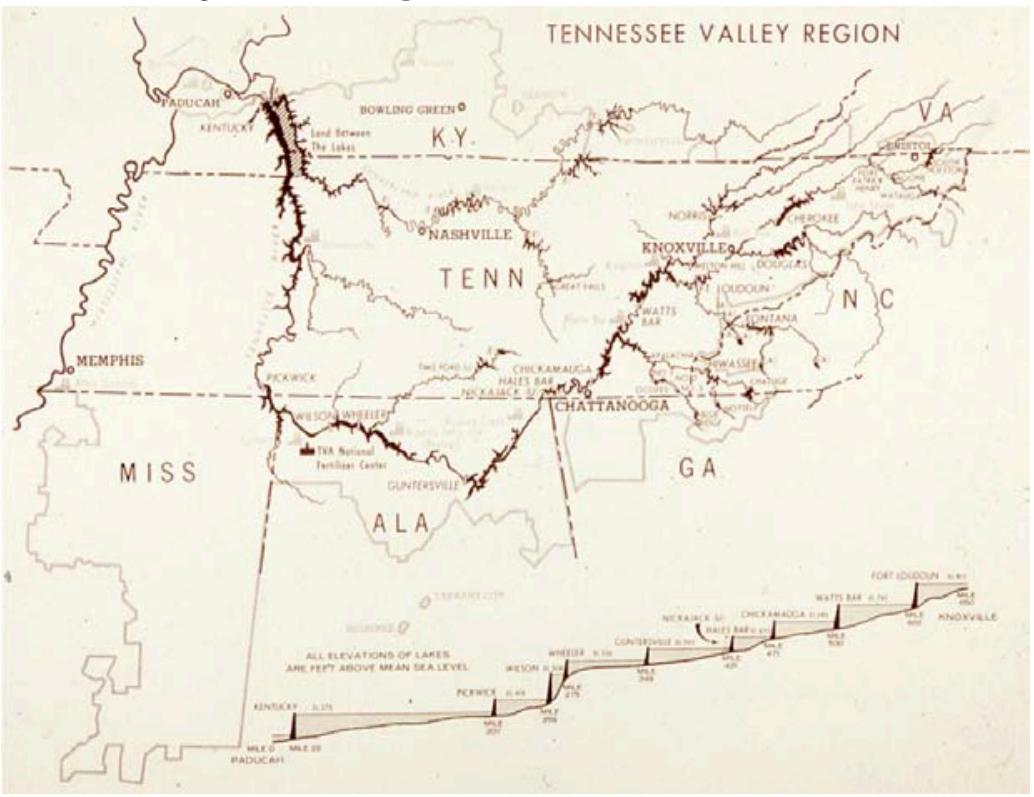

Fuente: Potenza (1975).

\section{Contexto}

Lo que tenía en común todo este territorio era la pobreza. Se trataba de la parte más pobre de la región más pobre de los EE.UU., con miles de familias subsistiendo con menos de 100 dólares al año. Esta área subdesarrollada disponía de una renta media inferior al 50\% de la renta media estadounidense, con la mitad de las familias recibiendo subsidios y donde tan sólo el $2 \%$ de las viviendas disponía de energía eléctrica. Se trataba de una región con graves desequilibrios interiores, además de un considerable retraso respecto al resto del país en términos de renta per cápita, de productividad, de energías empresariales y de equipamientos e infraestructuras. El territorio estaba en profunda regresión, ya que la única industria manufacturera, la maderera, estaba desforestando el bosque desde principios de siglo. La pérdida de la capacidad de almacenamiento de agua del bosque suponía una pérdida de recursos naturales contra las inundaciones. La erosión del suelo era enorme. Además, otro factor añadido era la imposibilidad de navegar por el río Tennessee por su caudal estacional y la falta de regulación de la propia cuenca.

\section{Objetivos}

Los objetivos, competencias y la fórmula jurídica de la TVA tenían importantes paralelismos con los del New Deal:

- Relanzar la política de inversiones.

- Establecer un control y una reglamentación de los recursos naturales de un área regional pluriestatal.

- Eliminar o disminuir el proceso de subdesarrollo de la cuenca. 
- Implementar un control y una reglamentación desde el estado de la distribución eléctrica y de sus tarifas.

- Reutilización del dique Wilson y de la fábrica de nitratos.

- Dotar al área progresivamente de infraestructuras e industrias.

- Potenciar y reestructurar los asentamientos y la actividad agrícola.

- Reorganizar los asentamientos residenciales-agrícolas para frenar el proceso de pérdida de población iniciado en los años 20.

- Realizar nuevas formas de control del proceso de desarrollo territorial por parte de la población del valle (democracia desde la raíz).

- Formular nuevas técnicas de planificación territorial.

Uno de los objetivos principales consiste en la recomposición del territorio y en la programación de un ciclo productivo completo mediante un sistema equilibrado y autosuficiente. De hecho, la realización de presas, la regularización del río y la puesta en marcha de centrales eléctricas deben considerarse como políticas de carácter territorial. Por tanto, desde la fertilización de los suelos a la implementación de todos los recursos, pasando por la construcción de los asentamientos, pueden ser concebidas como instrumentos para el logro de un equilibrio entre la ciudad y el campo capaz de contrarrestar el declive de la población en las áreas rurales y el crecimiento desordenado de los centros urbanos. En palabras de Lilienthal, es el primer experimento en la historia de América que considera conjuntamente, enlazándolos, tierra, agua, bosques, minerales, agricultura, industria y género humano.

\section{La intervención}

\subsection{Previsión de resultados}

En pocos años los resultados de las intervenciones de la TVA son espectaculares. Aparte de las dificultades con las empresas privadas, se implementan las distintas centrales eléctricas en función de la localización de las 8 presas más importantes: Gilbertville, Pickwick, Landing, Wilson, Wheeler, Guntersville, Hiwassee y Norris; que constituyen polos de referencia para las instalaciones menores (en total, la TVA construye 16 nuevas presas y reconstruye 5 más) (Droze, 1965) (ver Figura 3). 
Figura 3. Diagrama del sistema hídrico de la Tennessee Valley Authority

Fuente: Potenza (1975).

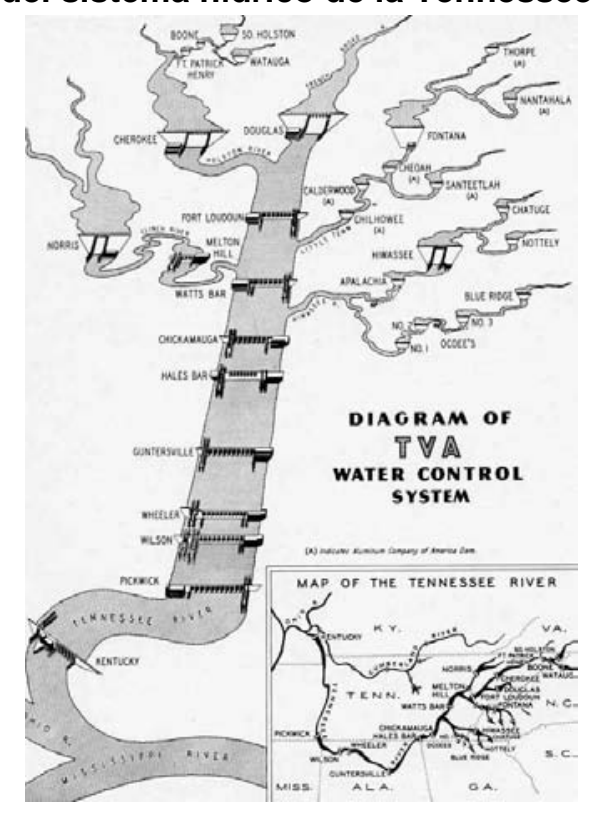

La presa Norris, una de las presas más importantes, fue realizada entre 1933 y 1936, con una longitud de más de 600 metros, una altura superior a los 80 metros y con una capacidad productiva de más de $50.000 \mathrm{kw}$. Su construcción supuso una inversión de más de 30 millones y medio de dólares.

\subsection{La electrificación}

La electrificación rural sufrió la siguiente evolución: 10\% de las granjas agrícolas en 1930; 40\% de las granjas agrícolas en 1940; y 90\% de las granjas agrícolas en 1950.

Esta mejora energética se traduce en un importante aumento sobre la producción agrícola e industrial, obligando a las compañías privadas a adaptarse a la nueva situación. La electrificación rural implicó la incorporación de nuevas industrias, la generalización del alumbrado eléctrico, la incorporación de las cámaras frigoríficas en las viviendas y, en general, un aumento significativo del confort doméstico. El servicio de electrificación tenía un alcance nacional. Por tanto, las repercusiones de esta intervención no afectaban exclusivamente a la zona del valle del Tennessee, sino que repercutían en un territorio muchísimo más amplio. En este sentido, el diagrama muestra el aumento de tarifa eléctrica en función de la distancia a la área productiva (ver Figura 4). 
Figura 4. Esquema de la tarifa eléctrica en función de la distancia

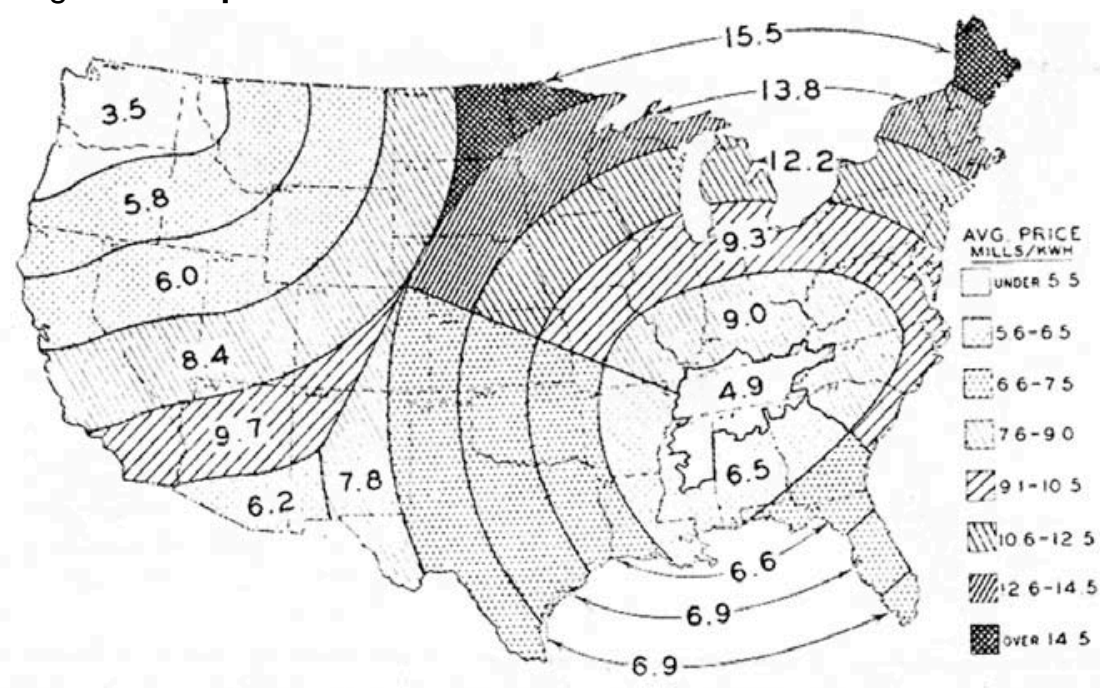

Fuente: Fonti (2005)

En el año 1944, esta zona se convirtió en la segunda productora de energía más grande de los EE.UU., generando en 1941 casi la mitad de toda la producción nacional. En conjunto, el valle resumiría todos los tipos de energías, desde las propias centrales hidroeléctricas, pasando por la energía solar, las turbinas de combustión, las centrales de carbón, la central térmica de Widows Creek o incluso el primer reactor nuclear del mundo.

\section{Los núcleos residenciales}

\subsection{Arthur Ernest Morgan y Norris Town}

Arthur Ernest Morgan, ingeniero de formación, deviene el líder de la planificación social, y supone el alma del nuevo asentamiento de Norris (Morgan, 1974). Morgan es de rigurosa observancia calvinista, y fuertemente influenciado por escritos sobre distintas colonias, por el texto La democracia de Carlyle y, en especial, por Edward Bellamy y su libro Looking Backward, una novela utópica en la que un joven americano se despierta en su misma localidad 30 años más tarde, en un entorno similar al socialismo utópico.

Para Morgan, la planificación social consiste en pequeños asentamientos donde poder equilibrar los distintos elementos de la comunidad: la vida social, política, personal y familiar (Talbert, 1987). En especial en asentamientos rurales. Bajo estos principios promovió proyectos de colonias industriales y planificó pequeñas ciudades que seguían los criterios de las garden cities inglesas. Entre ellos destaca la comunidad de Celo, fundada en 1937, con sus propias reglas de impuestos donde la tenencia de la tierra está controlada por la misma comunidad, donde no se requiere que sus miembros acepten cualquier religión o ideología, pero basada en los ideales de cooperación entre residentes y cuidado para el entorno natural. Hoy en día, Celo está formada por 40 familias que viven en una superficie de 1.200 acres, con un criterio cercano al de los quakers. 
Después de esta primera tentativa, y aprovechando la construcción de los primeros poblados para los trabajadores que se encargarían de la ejecución de las presas, se crearon distintos núcleos rurales de apoyo y desarrollo, y que posteriormente pasarían a ser asentamientos permanentes. El esquema organizativo de estos nuevos asentamientos consistía en un núcleo urbano de dimensiones acotadas rodeado por un anillo verde que entendían de protección, y que incorporaba a su vez áreas recreativas (ver Figura 5). Más allá de este anillo se distribuían de manera muy heterogénea pequeñas edificaciones vinculadas en algunos casos a la producción agrícola, como granjas y almacenes agrícolas, en otros casos edificios fabriles, o también áreas de ocio que requerían alguna edificación de servicios.

\section{Figura 5. Esquema organizativo de los núcleos urbanos}

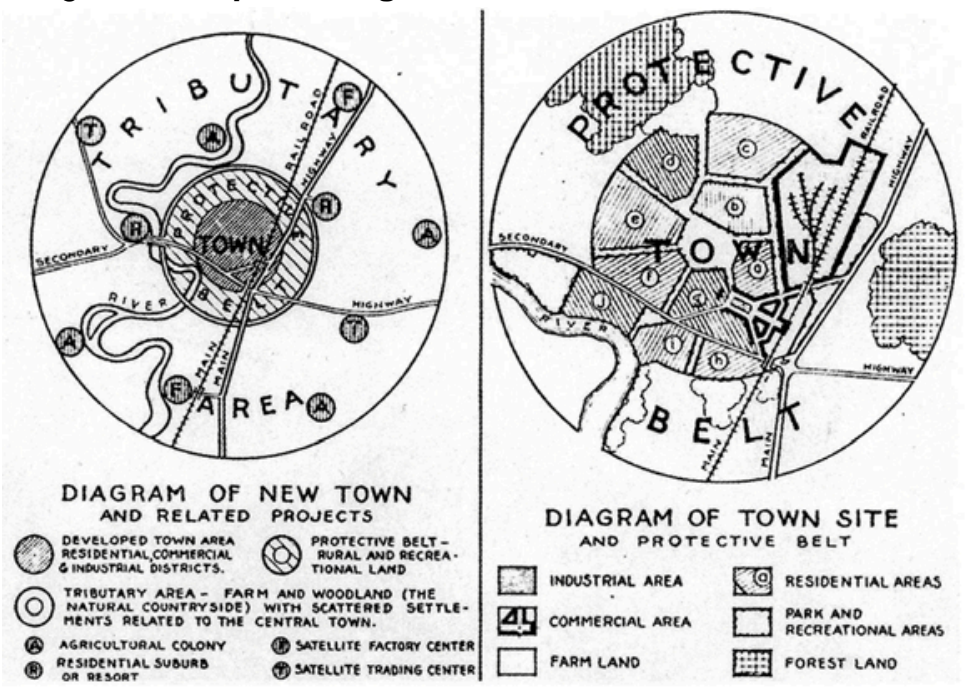

Fuente: Fonti (2005).

El núcleo urbano, que debía estar muy bien comunicado con el territorio, presentaba una estructura organizativa muy sencilla, basada en un pequeño centro comercial, unos comedores comunales, además de la escuela, la biblioteca, la oficina de correos y otros edificios públicos.

Morgan pidió al arquitecto Earle S. Draper para realizar el poblado de Norris, cerca de la presa de Norris. La lógica de este asentamiento se basa en aquella que ya se apuntó en las primeras colonias jardín inglesas, Port Sunlight y Bourneville: la eficiencia del trabajo del individuo viene determinada del modo en que éste pasa su tiempo libre (ver Figura 6). 
Figura 6. Planta del proyecto del núcleo urbano de Norris

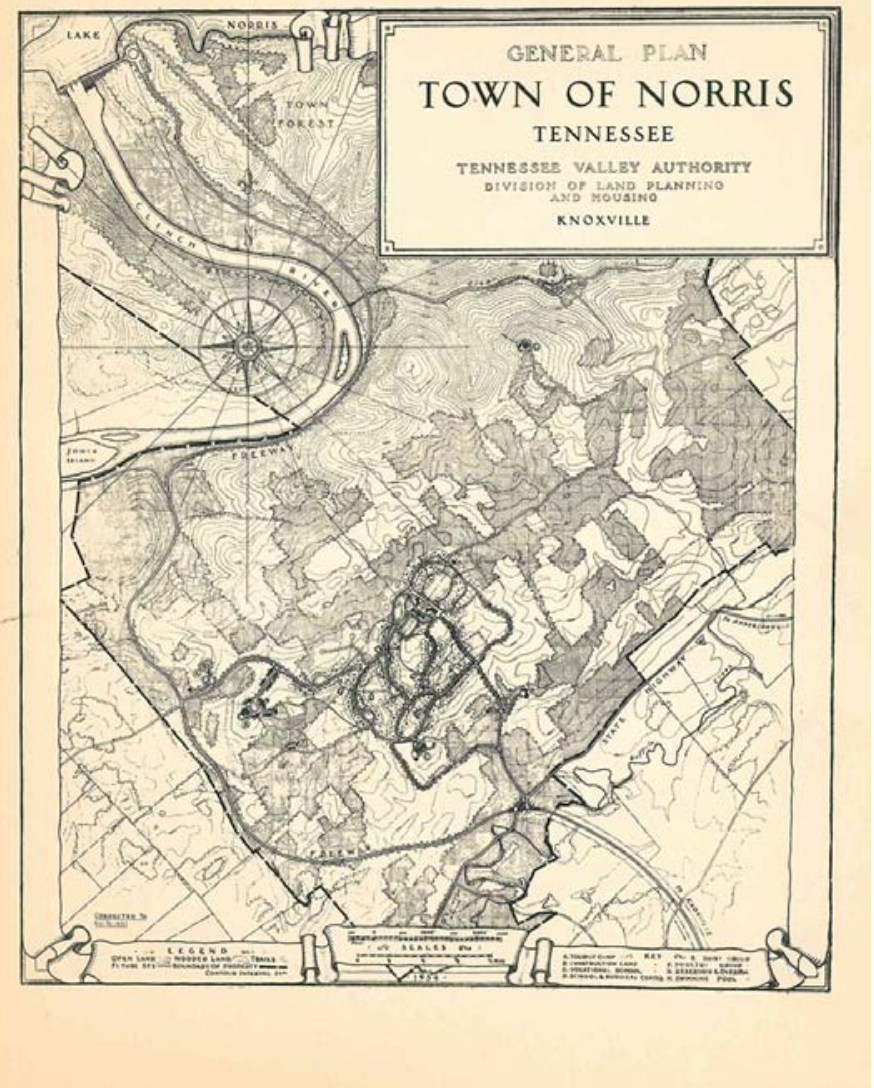

Fuente: Archivo Nacional de Atlanta.

El 5 de agosto de 1933 el consejo directivo del TVA autoriza la adquisición de los terrenos a 30 millas de Knoxville, cercana a la futura presa, aunque aislada de las conexiones infraestructurales. La construcción de Norris Town se inició en agosto de 1933 y se concluye en la primavera de 1935. Norris deviene así un lugar donde experimentar el nuevo orden social roosveliano donde la educación individual se mide por su capacidad de incidir en la actividad social y en la medida que puede evitar la degradación natural: en esto se basa el social planning, el alma que debía existir en el trasfondo de estas nuevas colonias (Grant, 1990).

Pero la nueva ciudad no debe ser tan sólo una ciudad modelo desde el punto de vista social, sino también un modelo de housing como símbolo de la intervención pública en la fase experimental de la planificación nacional. Es por ello que Draper se rodeó de planificadores progresistas, entre los cuales se encuentra Benton MacKaye, miembro destacado de la RPAA.

El asentamiento está construido sobre un relieve accidentado, y muestra un tejido extensivo, con un centro administrativo y escolar alrededor de un espacio libre y al servicio de los conjuntos de viviendas unifamiliares, y rodeado de un greenbelt exterior de protección, con un espacio abierto central. Su diseño responde por tanto claramente a las influencias de las 
ciudades jardín inglesas. El núcleo urbano está compuesto por 340 viviendas construidas sobre un ámbito de 1.600 hectáreas de densos bosques.

En el diseño del asentamiento las unidades residenciales se disponen a lo largo del trazado viario que se adapta a la configuración del relieve y del paisaje. No se realiza una parcelación previa, sino que la disposición de las viviendas viene determinada por los condicionantes escénicos del paisaje.

La ciudad fue presentada por Benton MacKaye como un primer paso en el desarrollo de la comunidad regional, o cómo definiría por el director de planificación del consejo del Valley, una ciudad rural. Tal y como ya indicó en su trabajo Employment and Natural Resources, la valoración que él mismo hace sobre Norris es muy favorable: por fin ve realizado un asentamiento con las características de sus working-units, que en este caso traduce como la unit of self mantenience. Dentro del plano regional, Norris tiene una función específica como comunidad permanente y que aporta riqueza al concepto regional: Norris Town debe funcionar como la sede del laboratorio de investigación y servicio de la organización territorial económica y social de las self mantenience units, dentro del sistema metropolitano de Knoxville y como centro residencial satélite.

\subsection{Las viviendas}

Las asignaciones presupuestarias eran muy limitadas, de manera que para abordar la construcción de los edificios residenciales se optó por utilizar edificios de bajo coste mediante prefabricados y elementos desmontables, para facilitar el transporte y reducir gastos. La idea de la prefabricación también supuso otras experiencias paralelas, como la construcción de viviendas trasladables. En conjunto, las viviendas pretenden ser una síntesis de la idea del New Deal, mediante una combinación entre la forma folk y la racionalidad progresista (ver Figura 7). La forma arquitectónica de los edificios es determinada por un estudio sobre la arquitectura tradicional del valle del Tennessee, que supone la elaboración de un reportaje fotográfico del sector y la recopilación de información acerca de las costumbres, los estándares de vida, etc. Por ello, la arquitectura no tiene grandes pretensiones. Interesa en este sentido sencillamente describir un asentamiento rural, donde se incentive el valor de la familia, de la comunidad rural con una fuerte tradición propia. Los materiales utilizados son principalmente la madera y la piedra. 
Figura 7. Una vivienda tipo en Norris Town

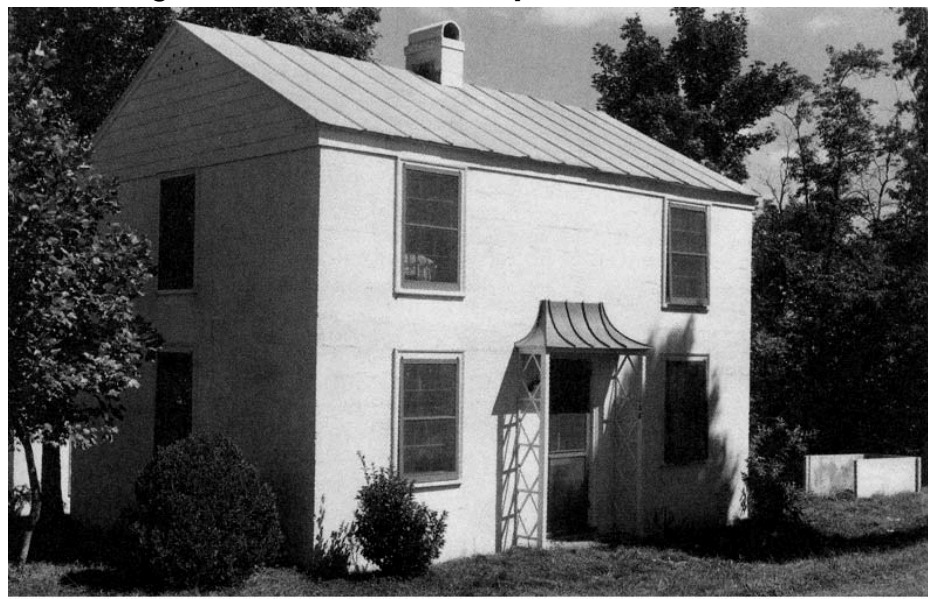

Fuente: Fonti (2005)

Se proponen en Norris más de 40 tipologías distintas, donde predominan las viviendas unifamiliares y bifamiliares, las casa en dúplex y los edificios de apartamentos para 8 familias. Se presta especial atención a la cocina, que denominan scientific kitchen, servida de todos los electrodomésticos necesarios; de hecho las primeras 150 unidades pretenden demostrar el uso de la energía producida por la presa Norris. Norris supone, así, un asentamiento ejemplar, que luego inspirará otras intervenciones del TVA, todas ellas cercanas a las presas: Wheeler, Pickwick Landing, Chickamauga, Hiawassee, Guntersville y Fontana.

\subsection{La idea de Henry Ford y Thomas Edison: la Seventy-Five Miles City}

A lo largo de todo este proceso, se produce un episodio de voluntades que merece la pena comentar, ya que supone una aportación singular desde la iniciativa privada con el objetivo de ordenar el territorio a partir de estos ideales de comunidad antes esbozados, a pesar de que el proyecto no llegara nunca a realizarse.

En el momento en que el gobierno federal está todavía construyendo la polémica presa de Muscle Shoals, en 1921, Henry Ford plantea al estado la compra de la presa para hacerse con el negocio, justo cuando ya se entrevé los enormes gastos de mantenimiento que el complejo ya empezaba a plantear en comparación con su efectividad. Henry Ford llega a Alabama en su tren particular, junto con su amigo Thomas Edison, el incomparable inventor y genio de la física capaz de transformar en luz la fuerza del agua. Ford ilustra su intención de crear en Muscle Shoals el mayor centro industrial del país, con una población de 1 millón de personas, construyendo una nueva ciudad lineal a lo largo del río de $400 \mathrm{~km}$, la famosa Seventy-Five Miles City (ver Figura 8), que estaría formada por varias ciudades importantes y otras de menor tamaño, de manera que sus residentes y sus familias vivirían en pequeñas comunidades donde los beneficios de vivir en entornos rurales serían perfectamente viables. 
Figura 8. La Seventy-Five Miles City

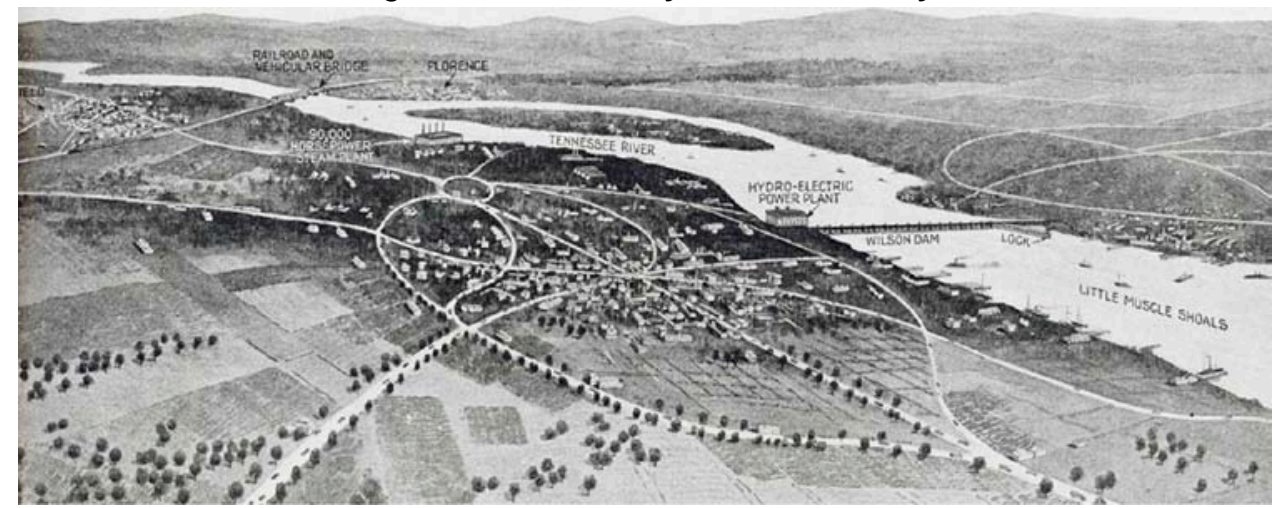

Nota: En la imagen se aprecia la presa Wilson, los núcleos urbanos preexistentes de Florende y Sheffield, y la idea de cómo se plantea el esquema general del nuevo sistema de ciudades).

Fuente: Fonti (2005).

En ella, cada uno de los trabajadores dispondría de una granja, de manera que debería compaginar los trabajos de la fábrica con los de la granja, permitiendo así establecer una economía de trabajo más equilibrada. De esta manera, un empleado sería un productor de alimentos y un asalariado en la fábrica al mismo tiempo, donde la fábrica de fertilizantes y de energía eléctrica funcionaría de manera conjunta y paralela con las granjas. Las expectativas de Ford llegaban a plantear una nueva ciudad que rivalizara con Detroit, la ciudad productora de automóviles por excelencia, asegurando que quedaría en un lugar secundario ante esta nueva ciudad regional.

\section{Las actividades complementarias}

\subsection{Las áreas recreativas}

Actualmente Norris es una ciudad dormitorio que depende de Knoxville, habitado por una clase media de funcionarios de la TVA. Incorpora una escuela elemental hasta los 5 años y una escuela media de 6 a 8 años. Pero, como ya hemos comentado, desde los inicios el diseño del núcleo urbano muestra inquietudes por la actividad comunitaria, que se traduce también en la construcción de áreas de ocio, como el Big Ridge State Park, un complejo turístico con numerosos campings.

A partir de esta idea de incorporar áreas lúdicas con fines recreativos acordes con la búsqueda de la vida comunitaria que fomente la interrelación social, miles de hectáreas de tierra se transforman en parques públicos administrados por la misma TVA o transferidos a la administración de los estados o de los condados (Dalco, 1975).

La construcción de las presas supuso la aparición de enormes lagos que se convirtieron en el espacio recreativo de miles de ciudadanos que podrán a partir de entonces pescar, navegar y, en definitiva, disfrutar de estos nuevos espacios, que supondrían la aparición de nuevos centros turísticos y campamentos que se emplazaron alrededor de los lagos. Las inversiones 
aseguraron la navegabilidad en más de $1.000 \mathrm{~km}$ de recorrido, y supusieron la creación de más de $15.000 \mathrm{~km}$ de playas para el recreo de la población a lo largo de las orillas de los ríos y embalses. El área recreativa de Land Between The Lakes supone una zona lúdica de alcance nacional, que utiliza el recurso hídrico como argumento principal de las amplias zonas recreativas, y es una de las áreas más significativas de nueva creación. También destacan la Barckley Lodge, el Lake Chickamauga o la Chuck Swan Wildlife Management Area, entre otras (ver Figura 9). 
Figura 9. El parque recreativo del Land Between the Lakes

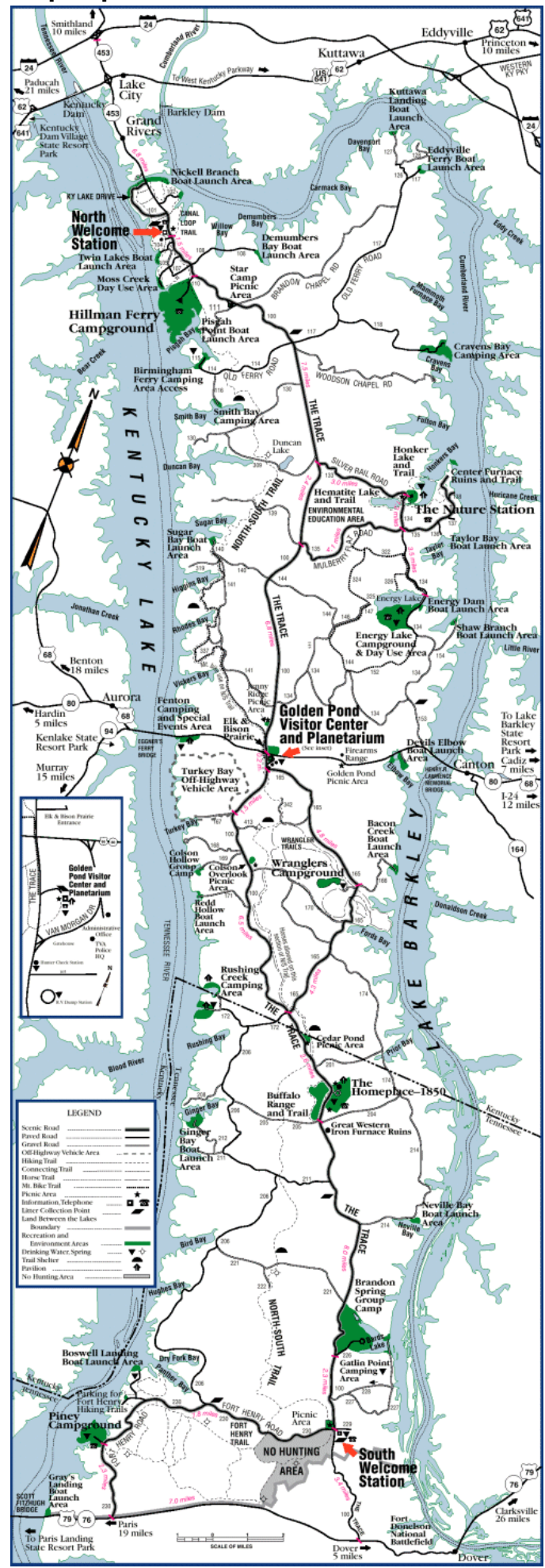

Fuente: Bits \& Pieces (2007). 


\subsection{La agricultura y otros réditos}

La fuerte experimentación agrícola con el uso de fertilizantes ha sido también motivo de atracción de visitantes y expertos en la materia de todo el mundo. Un promedio de 2.000 técnicos de 70 países visitaron anualmente el área del TVA en los años 50.

La tierra sin cubierta vegetal estaba a expensas de la erosión. Las nuevas instalaciones construidas junto a las presas fueron capaces de producir 150.000 toneladas de fosfatos al año utilizando los yacimientos de la zona media del río, que se utiliza para la revitalización de los terrenos agrícolas, justo en el momento en que las fincas pueden disfrutar de energía eléctrica barata y aprovechar las oportunidades de asesoramiento que les ofrece el ente público para la adecuación de sus técnicas de producción. El cuidado por las explotaciones agrícolas y las granjas repercutió en un trabajo importante de investigación sobre los fertilizantes, e incorporó incluso visitas guiadas a las instalaciones. La preocupación por los fertilizantes conllevó la construcción de diversas plantas productoras de nitratos y de fosfatos, como las plantas de Liberty Bells.

La intervención de la TVA supuso también significativas mejoras en otros aspectos, en especial en las infraestructuras: se construyen más de $200 \mathrm{~km}$ de nuevas carreteras y $225 \mathrm{~km}$ de vías férreas. Además, decenas de miles de trabajadores quedan empleados en las obras de reforestación, de manera que en las riberas que dan a las presas y los embalses se colocan más de 20 millones de plantas. El patrimonio piscícola se multiplica por 40. Las repercusiones también son de carácter cultural, ya que aparecen paralelamente distintos museos, como el museo de los Apalaches, el museo de la Ciencia y de la Energía, el museo Infantil o la creación del arboretum de la Universidad de Tennessee.

\section{Conclusión}

A pesar de la dura oposición de los intereses más conservadores, encasillados en los principios del liberalismo económico integral, la TVA desarrolla su programa hasta el final. De hecho, la TVA representa el éxito más notable del New Deal en el campo de la planificación, y en los años de posguerra sigue siendo un importante punto de referencia para los teóricos del Welfare State. En este sentido, supuso el empleo directo para 200.000 personas, la creación de distintas iniciativas pioneras en otros sectores, el aumento de las rentas y consumos respecto otras regiones, con un balance financiero positivo. La TVA demuestra por tanto el buen resultado de una ideología del intervencionismo público programado, con un esfuerzo gigantesco y resultados no menos importantes, y que suponen al final, por los métodos y las estructuras organizativas, la resurrección y reestructuración del nuevo capitalismo.

Sin embargo, los grupos conservadores del Congreso y los intereses privados no aparecerán dispuestos a permitir ninguna aplicación de esta experiencia, bloqueando el programa del presidente en el año 37 para la creación de 7 nuevas agencias federales similares a la TVA, algunas para la costa atlántica, la costa pacífica, para los Grandes Lagos y para el valle de Ohio. En este sentido, es bien representativo el destino de la ciudad de Norris de hasta qué punto llegaban las presiones de los conservadores y los privados, ya que la ciudad se puso a la venta en subasta pública en 1948, con todo su patrimonio edificado, las instalaciones 
hidráulicas, el alcantarillado, los equipamientos colectivos y los edificios escolares, detrás de una fracasada tentativa de las familias residentes a asociarse y adquirir por sí mismas la ciudad, ya que sus posibilidades financieras quedan muy por debajo de las ofertas presentadas por un grupo privado de Filadelfia.

De todas maneras el experimento de TVA no se podría repetir, ya que no permitía la libre competencia por ejemplo en la industria eléctrica, y tras una nueva crisis entre 1937 y 1938 aparecieron serias dificultades en los poderes suprafederales. Además, los holdings eléctricos lo han visto siempre como un mecanismo de control del precio de la energía, y se opusieron a la repetición del modelo ya que lo juzgaban anticonstitucional. Sin embargo, el TVA ha sido un modelo de referencia en el debate sobre los instrumentos de gestión urbanística (público/privado) y muy especialmente por su escala de intervención.

Efectivamente, las intervenciones posteriores vinculadas a otros ámbitos de cuenca no disfrutaron de la exclusividad y el control que la TVA tuvo en todo momento. Todas las decisiones eran en este sentido definitivas, en la medida en que los distintos departamentos gubernamentales, como el de agricultura, el hidroeléctrico, el de medio ambiente o el de ingeniería, por citar algunos, dependían de las decisiones tomadas en el seno del organismo de la TVA y de su propia autoridad. Las intervenciones que se llevarán a cabo tras la experiencia del TVA, como por ejemplo las implicadas en el río Columbia, no iban a tener competencias exclusivas. No había una autoridad única, sino que las propuestas iban a depender de la lectura y de la aprobación de los diferentes departamentos implicados, limitando así la capacidad de decisión y gestión de la iniciativa. A pesar de ello con la inauguración de la gran presa de Coulee Dam en el río Columbia en 1942 se mostraba la que durante muchos años sería la mayor y más avanzada presa del mundo y modelo de las futuras grandes presas africanas y asiáticas. En Gran Coulee toda la energía producida se dedicaba a elevar agua para irrigar un inmenso valle.

En cualquier caso, el resultado final de la TVA, en cuanto a cifras se refiere, supone ser el mayor suministrador eléctrico de los EE.UU., con 155.000 millones de Kw al año. La TVA trabaja en coordinación con 159 distribuidores locales que suministran a 9 millones de personas. La TVA tiene 11 plantas térmicas, 5 de combustión-turbina, 29 hidroeléctricas, 3 de energía nuclear y una estación de bombeo para el reciclado de energía sobrante. Así mismo cuenta con nuevos centros de energía solar, eólica y de gas metano. Además, la TVA gestiona 164 áreas de recreo y el Land Between The Lakes, que como hemos comentado es de carácter nacional. Se estima que los visitantes aportan anualmente a la economía del valle unos 1.260 millones de euros. 


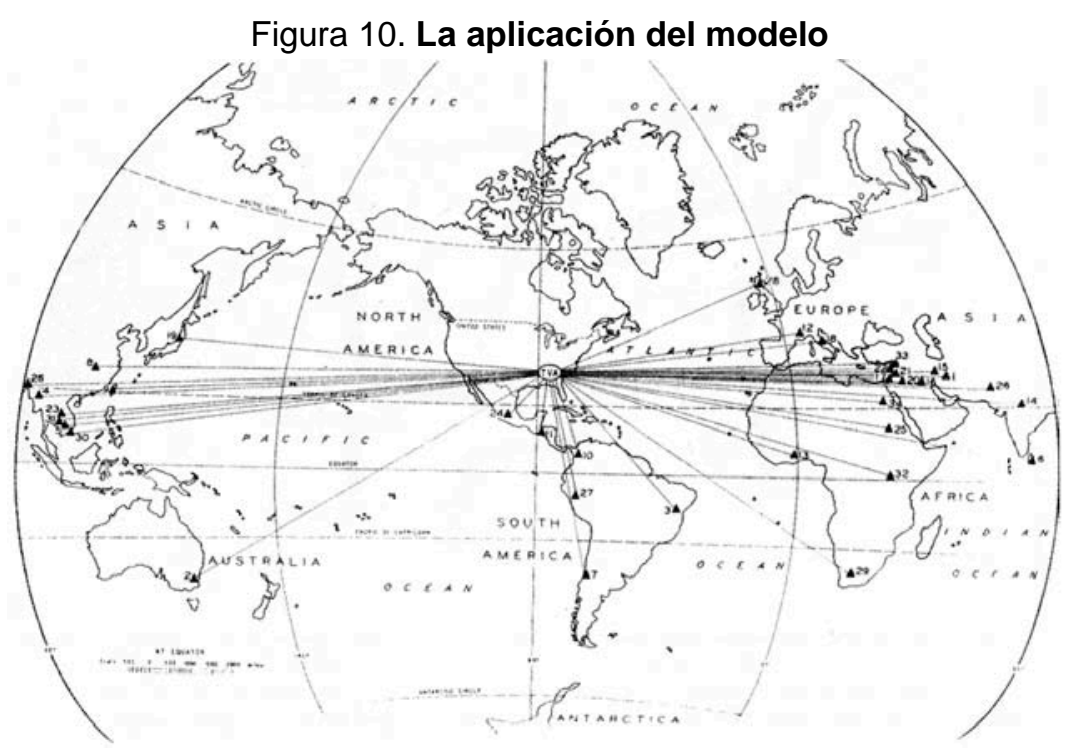

Fuente: Fonti (2005).

La Figura 10 muestra la aplicación del modelo TVA en el mundo ya en 1973. El número 18 corresponde a la intervención italiana de la Cassa per il Mezzogiorno; el 34 corresponde a la presa de Asuán. Pero sin duda alguna, la aplicación más significativa de este modelo es la presa de las tres gargantas en el río Yangtzé. Esta construcción supone la planta generadora de electricidad mayor del mundo.

La experiencia de la Tennessee Valley Authority supone, por tanto, un importantísimo referente en la planificación territorial, tanto en términos de su repercusión en el ámbito físico de su intervención como en términos de su repercusión nacional, en la medida que implica una reactivación y estabilización económica del país, aportando al mismo tiempo repercusiones energéticas, productivas, culturales, lúdicas, y también de colonización del territorio mediante nuevos asentamientos urbanos.

\section{Bibliografía}

BITS \& PIECES. Fecha de consulta: 17 de diciembre de 2012. Disponible en: <http://bitsandpieces1.blogspot.com.es/2007_07_08_archive.html>. 2007.

CORLEW, R.E. Tennessee. A Short History. Knoxville, The University of Tennessee Press, 2003. $636 \mathrm{p}$.

CREESE, W. TVA's Public Planning. Knoxville, University of Tennessee Press, 1990. 388 p.

DALCO, F. De los parques a la región. En: La Ciudad Americana. De la guerra civil al New Deal, AAVV, 1975, GG, Barcelona, pp: 162-165. 
DROZE, W.H. et al. TVA Rebuilds a River. Los Ángeles, Louisiana State University Press, 1965. $174 \mathrm{p}$.

FONTI, A. Norris Town 1933-38: architettura e pianificazione nella Tennessee Valley Authority. Milán, Metodi del Territorio, 2005. 112 p.

GRANT, N.L. TVA and Black Americans: Planning for the Status Quo. Philadelphia, Temple University Press, 1990. 207 p.

HARGROVE, E.C. y CONKIN, P.K. (Eds.) TVA: Fifty Years of Grass Roots Bureaucracy. Champaign, University of Illinois Press, 1984. 432 p.

HUXLEY, J. TVA Adventure In Planning. ED: The Architectural Press. Londres, 1943. 142 p.

KYLE, J.H. The Building of TVA: An Illustrated History. os Ángeles, Louisiana State University Press, 1958. $162 \mathrm{p}$.

MOORE, J.R. (Ed.) The Economic Impact of TVA. Knoxville, University of Tennessee Press, 1967. $163 \mathrm{p}$.

MORGAN, A.E. The making of TVA (1 $1^{\mathrm{a}}$ Ed.). New York, Prometheus Books, 1974. 205 p.

POTENZA, S. L'urbanistica dil riformismo USA 1890-1940. Milán, Mazzotta, 1975. 236 p.

TALBERT, R. FDR's Utopian: Arthur Morgan of the TVA. Jackson, University Press of Mississippi, 1987. $244 \mathrm{p}$. 
Architecture, City, and Environment

Arquitectura, Ciudad y Entorno

ACEO AÑO 10, núm.28, JUNIO 2015|TENNESSEE VALLEY AUTHORITY: UNA EXPERIENCIA DE PLANIFICACIÓN TERRITORIAL MODÉLICA 